\title{
Prevalencia de diabetes gestacional en el Hospital Gestionar Bienestar, Zapatoca, Santander 2013 - 2017
}

Jorge Leonardo Vergara Camargo*

*Médico general. Hospital Gestionar Bienestar. Zapatoca. Santander. Colombia. Correspondencia: Dr. Jorge Vergara. Dirección: Carrera 2 \# 22-30, barrio La Merced, Zapatoca, Colombia. Correo electrónico: qtzleonardo@gmail.com Celular: (+57) 30175812098

Resumen

Introducción: la diabetes gestacional se define como la hiperglucemia con inicio o primer reconocimiento durante el embarazo. Es una de las complicaciones más comunes del embarazo, con una prevalencia de hasta el 10\% en los países desarrollados y con limitada información en población colombiana. Objetivo: determinar la prevalencia de diabetes gestacional y la frecuencia de los factores de riesgo en pacientes que acudieron al Hospital Gestionar Bienestar de Zapatoca, Santander, entre 2013 y 2017. Materiales y Métodos: se realizó un estudio descriptivo, retrospectivo de corte transversal, se trabajó con una muestra de historias clínicas de pacientes que acudieron a controles prenatales al Hospital de Zapatoca, y que culminaron sus gestaciones entre junio 2013 y abril 2017. Resultados: de 297 gestantes registradas, 269 fueron incluidas para determinar la prevalencia de diabetes. En promedio, las participantes empezaron sus controles con $12 \pm 0,74$ semanas de gestación y edad de $25 \pm 0,7$ años. La prevalencia de diabetes gestacional fue de $4,46 \%$. El $41,32 \%$ de las gestantes empezó controles prenatales con sobrepeso u obesidad según clasificación del estado nutricional de Atalah, encontrándose mayor frecuencia de diabetes en las gestantes con elevado índice de masa corporal y edad materna avanzada. Conclusiones: la prevalencia obtenida en el presente estudio es inferior a la descrita en otros estudios en Latinoamérica. Los resultados sugieren que la edad materna avanzada y el índice de masa corporal alto al inicio de la gestación fueron factores de riesgo para desarrollar diabetes gestacional en esta población. MÉD.UIS. 2018;31(2):17-23.

Palabras clave: Diabetes Gestacional. Prevalencia. Factores de Riesgo. Sobrepeso. Obesidad

\section{Prevalence of gestational diabetes in the Gestionar Bienestar Hospital, Zapatoca, Santander 2013-2017}

Abstract

Background: gestational diabetes is defined as hyperglycemia with onset or first recognition during pregnancy. It is one of the most common pregnancy complications, with a prevalence of up to $10 \%$ in developed countries and with limited information in colombian population. Objective: to determine the prevalence of gestational diabetes and the frequency of risk factors in patients who attended the Gestionar Bienestar Hospital of Zapatoca, Santander, between 2013 and 2017. Materials and Methods: a descriptive retrospective crosssectional study was carried out. A clinical sample of patients who came to perform their prenatal checkups at the Gestionar Bienestar hospital of Zapatoca, who completed their pregnancies between june 2013 and april 2017. Results: of 297 registered pregnant women, 269 were included to determine the prevalence of diabetes. On average, the participants started their controls with $12 \pm 0.74$ weeks of gestation and age of $25 \pm 0.7$ years. The prevalence of gestational diabetes was $4.46 \% .41 .32 \%$ of the pregnant women started prenatal controls with overweight or obesity according to Atalah nutritional status classification, with higher frequency of diabetes in pregnant women with high body mass index and advanced maternal age Conclusions: the prevalence obtained in the present study was lower than that described in other studies in Latin America. The results suggest that advanced maternal age and high body mass index at the beginning of gestation were risk factors for developing gestational diabetes in this population. MÉD.UIS. 2018;31(2):17-23.

Keywords: Diabetes, Gestational. Prevalence. Risk Factors. Overweigh. Obesity 
¿Cómo citar este artículo?: Vergara JL. Prevalencia de diabetes gestacional en el Hospital Gestionar Bienestar, Zapatoca, Santander 2013 - 2017. MÉD.UIS. 2018;31(2):17-23. Páginas. DOI: 10.18273/ revmed.v31n2-2018002

\section{Introducción}

La diabetes gestacional se define como la alteración de la tolerancia a la glucosa de gravedad variable, detectada durante la gestación independientemente de la necesidad de tratamiento insulínico o de que continúe después del parto y que no excluye la posibilidad de existencia anterior no observada'. Esta definición generada en 2007 durante la Quinta Conferencia Internacional de Diabetes Gestacional conserva aún plena validez siendo solo resumida en "cualquier grado de intolerancia a la glucosa con inicio o primer reconocimiento durante el embarazo" por múltiples autores incluyendo la Asociación Americana de Diabetes (ADA, por sus siglas en inglés $)^{2-5}$.

La diabetes gestacional se ha reportado aproximadamente en el 3-10\% de los embarazos en los países desarrollados como Estados unidos ${ }^{6}$ con frecuencias de hasta $20 \%$ en países en vía de desarrollo como Bermuda y $\mathrm{Nepal}^{7}$, y en Latinoamérica, según la guía de práctica clínica Colombiana, la prevalencia varía entre $4,2 \%$ y $7,6 \%$,

Por otro lado, se ha descrito que las prevalencias reportadas varían incluso de acuerdo con la composición étnica de cada país al ser más frecuente en afrodescendientes, latinas y mujeres del sudeste asiático respecto a la mujer caucásica ${ }^{10}$. Según la guía antes mencionada, generada por el ministerio de protección social en 2015, no hay estudios de prevalencia, tampoco sobre la carga de la enfermedad en términos de mortalidad, morbilidad y pérdida de años de vida saludable9. Sin embargo, es posible encontrar estudios que sugieren una prevalencia de $6,3 \%$ en una población de Manizales en el $2014^{11}$ y de $7,5 \%$ para una población de Bogotá en el 2000, este último por García y colaboradores ${ }^{12}$, que citan cifras de la Asociación Latinoamericana de Diabetes $^{13}$. Quienes recientemente, junto con otros autores, advierten de que se trata de una condición que va en aumento ${ }^{14,15}$ debido fundamentalmente a una mayor frecuencia de obesidad en la edad fértil ${ }^{16}$.

La intolerancia a la glucosa como génesis de la diabetes gestacional tiene ocasión por una modificación en el metabolismo de los carbohidratos que condiciona una reducción de la sensibilidad a la insulina ${ }^{10-17}$. Más específicamente el defecto consiste en una alteración en la capacidad de adaptarse al incremento de la resistencia a la insulina fisiológica en el embarazo ${ }^{10,15,18,19}$, secundaria al efecto diabetógeno de algunas hormonas a partir del segundo trimestre de gestación ${ }^{20}$, como lo son el lactógeno placentario, la hormona placentaria del crecimiento y prolactina ${ }^{18}$.

El fenómeno de resistencia a la insulina llega a representaren un embarazonormal aproximadamente un 50\% de disminución en la disponibilidad de glucosa mediada por insulina y hasta un $200 \%$ de incremento en la secreción de insulina para tratar de mantener euglicémica a la madre ${ }^{18}$.

Existe amplia literatura sobre los factores de riesgo para desarrollar diabetes gestacional los cuales incluyen $7,9,15,18,21,22$; edad materna avanzada (OR 3,0 con IC $1,73-5,19)^{21,22}$, historia familiar de diabetes mellitus (OR 1,86 con IC 1,29-2,77) $)^{21,22}$, antecedente de diabetes gestacional, sobrepeso y obesidad (OR 10,1 con IC 5,61-18,39) ${ }^{21,22}$, antecedente de feto macrosómico (OR 2,08 con IC 1,52-2,86) ${ }^{21,22}$ y grupo étnico.

La diabetes gestacional trae consigo un incremento en la morbilidad materna y fetal, guardando una relación estrecha entre los niveles de glucemia materna y los resultados adversos, aunque no se ha descrito un umbral de glucemia en función del riesgo de cada complicación ${ }^{23}$.

Las complicaciones maternas más frecuentes que se han descrito son los trastornos hipertensivos del embarazo, la mayor tasa de cesáreas, parto pretérmino, polihidramnios y mayor probabilidad de desgarros durante el parto vaginal ${ }^{24-26}$. Respecto a los efectos maternos a largo plazo, se ha advertido una mayor probabilidad de desarrollar diabetes mellitus tipo 2 en las pacientes que padecieron diabetes gestacional, pues, aunque esta condición resuelve generalmente después del parto ${ }^{27,28}$, entre el $15 \%$ y el $50 \%$ de las gestantes progresan a diabetes tipo 2 en la siguiente década ${ }^{29-32}$.

A nivel de producto las complicaciones en orden de frecuencia son la macrosomía fetal, hipoglicemia neonatal, hiperbilirrubinemia, recién nacido grande para la edad gestacional, distocia de hombros y 
la muerte perinatal $1^{1,24,33,34}$, y a largo plazo existe evidencia de que el ambiente intrauterino será determinante en su predisposición a obesidad, diabetes mellitus tipo II, enfermedad cardiovascular y síndrome metabólico en la edad adulta ${ }^{32,35-37}$.

Estos importantes efectos deletéreos en el recién nacido y en la madre resaltan la importancia de focalizar la atención en la diabetes gestacional por lo que el objetivo de este estudio es determinar la prevalencia de diabetes gestacional en pacientes de un municipio colombiano, a la vez que se describen las características sociodemográficas y la frecuencia de factores de riesgo en la misma población.

\section{Materiales y métodos}

Se realizó un estudio de corte transversal, de tipo descriptivo y retrospectivo, donde se incluyeron todas las historias clínicas de gestantes que acudieron a sus controles prenatales al Hospital Gestionar Bienestar de Zapatoca, Santander y que culminaron la gestación (incluyendo partos, abortos y muertes perinatales) en la institución entre junio 2013 y abril 2017. Sin hacer distinción por edad materna o tipo de afiliación a seguridad social.

Se excluyeron las gestantes con diagnóstico previo de diabetes mellitus tipo 2 y aquellas de las cuales no se pudo continuar seguimiento de sus controles prenatales en la institución por cambio de residencia o de afiliación a entidad promotora de salud con subsecuente cambio de institución prestadora de salud durante el periodo en estudio. No se excluyeron las gestantes que antes de la semana 23 culminaron su gestación, para el estudio de las variable sociodemográficas en el primer control prenatal, pero si lo fueron para calcular la prevalencia de diabetes gestacional. Cada gestación se consideró una oportunidad para adquirir diabetes por lo que fue medida como caso independiente durante el periodo en estudio.

De manera sistemática se revisó la historia clínica completa de las gestantes incluyendo los controles prenatales por medicina general y las valoraciones por medicina especializada. En todos los casos el personal de enfermería adscrito a promoción y prevención recolectó información por vía telefónica a las participantes sobre el desenlace de la gestación cuando este se produjo en una institución diferente, esta última actividad de carácter protocolario para el cierre de la historia materna.
En todas las historias se observó el empleo de los criterios del Colegio Americano de Obstetras y Ginecólogos (ACOG, por sus siglas en inglés) planteados en 2013 para el diagnóstico de la diabetes gestacional, evaluándola en dos momentos, en primer lugar mediante tamizaje entre las semanas 24 y 28 de gestación con una carga de $50 \mathrm{~g}$ de glucosa; de cursar con alteración, se indica la realización de una segunda prueba de tolerancia con una carga de $100 \mathrm{~g}$ de glucosa con los siguientes puntos de corte para definir alteración: $>95 \mathrm{mg} / \mathrm{dl}$ en ayunas, $>180 \mathrm{mg} / \mathrm{dl}$ una hora después de administrada la carga, $>154 \mathrm{mg} / \mathrm{dl}$ dos horas después y $>140 \mathrm{mg} / \mathrm{dl}$ tres horas después.

Adicionalmente, se observó que desde el segundo semestre del 2016 se emplearon en la institución de manera simultánea a los criterios antes mencionados los propuestos por los grupos de estudio de la Asociación Internacional de Diabetes en 2010 (IADPSG, por sus siglas en inglés) adoptados por la Asociación Americana de Diabetes en 2011, que consisten en una única prueba de tolerancia entre la semana 24 y 28 , con carga oral de $75 g$ en ayunas con medición previa a la carga, a la hora y dos horas post carga; en este caso el diagnóstico se hace si se obtiene al menos un valor alterado, con los siguientes puntos de corte: $>92 \mathrm{mg} / \mathrm{dl}$ en ayuno, $>180 \mathrm{mg} / \mathrm{dl}$ a la hora y $>153 \mathrm{mg} / \mathrm{dl}$ a las 2 horas $^{38}$. Ambos métodos fueron considerados válidos para realizar el diagnóstico que en todo caso fue confirmado con la interpretación de los resultados en el control programado por ginecología entre la semana 30 y 34 de gestación.

Para la clasificación según estado nutricional se empleó la escala de Atalah validada para gestantes con más de diez semanas de gestación ${ }^{39}$, y en aquellas maternas que iniciaron controles con edades gestacionales menores, se emplearon los criterios propuestos por el comité de estado nutricional durante el embarazo del Instituto de Salud Estadounidense ${ }^{40}$.

Se obtuvo de las historias clínicas información acerca de las variables edad, residencia, escolaridad, antecedente previo de diabetes gestacional, Índice de masa corporal (IMC) al inicio de los controles, vía de parto, presencia de parto pretérmino, desarrollo de trastorno hipertensivo durante el embarazo y muerte perinatal. La información fue procesada en los programas EPI INFO 7 y Microsoft Excel. Se reportaron las frecuencias de todas las variables 


\section{Vergara JL}

estudiadas con límites de confianza del 95\% y medidas de tendencia central para las variables cuantitativas continuas. Solo se estratificó mediante cuatro intervalos la edad materna de la siguiente manera: $\leq$ 17 años, 18 a 26 años, 27 a 36 años $y \geq 37$ años.

\section{Resultados}

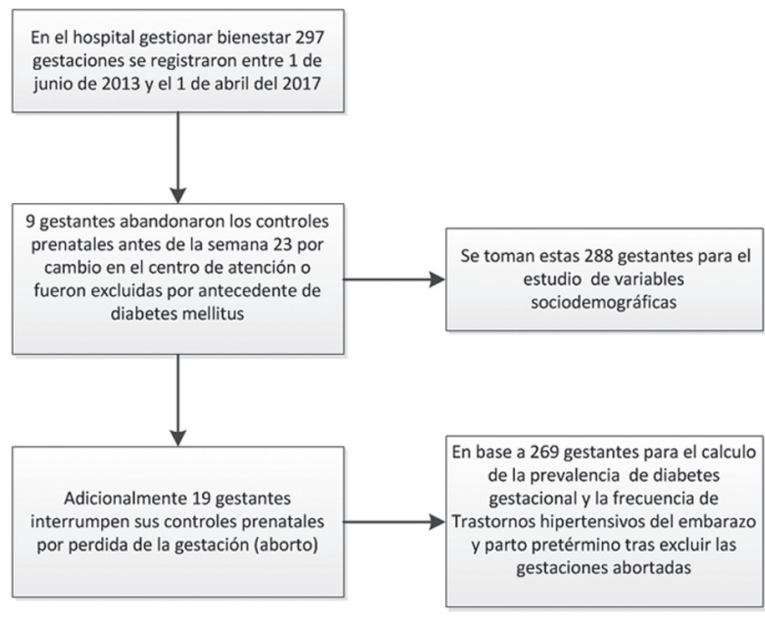

Figura 1: formación de la muestra

Fuente: autor

En total 297 gestaciones se registraron entre el 1 de junio de 2013 y el 1 de abril del 2017, de estas, nueve gestantes abandonaron el programa antes de la semana 23 por cambio en el centro de atención o fueron excluidas por antecedente de diabetes mellitus tipo I o II (Ver Figura 1). Las gestaciones cuyo desenlace fue aborto se excluyeron del cálculo de prevalencia, pero no para el estudio de variables sociodemográficas al primer control prenatal. Ninguna gestante cesó sus controles luego de la semana 24 por causas diferentes a la atención del parto en institución de mayor nivel de complejidad.

El máximo número de gestaciones presentadas por la misma mujer durante el periodo en estudio fue de dos en 25 participantes, pero de estas ninguna fue diagnosticada con diabetes gestacional; la prevalencia de diabetes gestacional fue $4,46 \%$ (IC al $95 \% 2,03-6,89$ ). El 3,13\% de las gestantes ya contaba con antecedente personal de diabetes gestacional. En promedio las gestantes empezaron sus controles con $12 \pm 0,74$ semanas de gestación y con una edad también promedio de $25 \pm 0,7$ años. El intervalo de edad que con mayor frecuencia incluyó pacientes con diabetes gestacional fue entre 27 y 36 años con un 58,3\% (IC al 95\% 27,67-84,83) de los casos.
MÉD.UIS. 2018;31(2): 17-23

La talla y peso promedio al primer control fueron $1,56 \pm 0,71 \mathrm{mts}$ y $63 \pm 1,63 \mathrm{~kg}$ respectivamente, el índice de masa corporal al primer control fue de $25,2 \pm 0,6$. El lugar de residencia más frecuente fue urbano correspondiendo al 52,4\%. La escolaridad de las gestantes en el $49.6 \%$ correspondió a secundaria completa, mientras un $10,7 \%$ se encontró cursando secundaria al momento del primer control prenatal y un $32,9 \%$ ya había interrumpido su formación académica secundaria antes de la gestación. Se encontró que el $23,3 \%$ de gestantes cursó con sobrepeso y el $18,06 \%$ con obesidad para el primer control prenatal. Específicamente, en la población diagnosticada con diabetes gestacional el sobrepeso representó el 50\% (IC al 95\% 21,09-78,91) y la obesidad el 33\% (IC al 95\% 9,92-65,11). Con distribución similar de vía de parto entre vaginal y cesárea con $43,7 \%$ y $49.6 \%$, respectivamente. (Ver Tabla 1 )

Finalmente, otras variables estudiadas como el desenlace mediante parto prematuro y el desarrollo de algún trastorno hipertensivo asociado al embarazo se encontraron frecuencias de $3,72 \%$ y $4,83 \%$, respectivamente (Ver Tabla 2 ).

\section{Discusión}

La prevalencia obtenida de $4,46 \%$ para diabetes gestacional es inferior a la descrita por Burbano ${ }^{11} \mathrm{y}$ García $^{12}$ en otros estudios nacionales, realizados en población predominantemente urbanas (superior a $90 \%$ en cada estudio). Zapatoca, un municipio con 8929 habitantes según cifras aproximadas del DANE para el 2015, está compuesto en un 60\% por población urbana, y solo un hospital que tiene a su cargo la atención de la población de dos de las tres entidades promotoras de salud principales en el municipio, que representan más del $70 \%$ de la población gestante, por lo que considera un acercamiento a la distribución de las variables estudiadas en una población pequeña colombiana, con un importante componente rural y el mayor tamaño muestral posible en el único hospital en el municipio durante el periodo en estudio.

Respecto a las cifras de referencia para otros países latinoamericanos, se encontraron prevalencias de entre $8 \%$ y $12 \%$ para México ${ }^{41} 12,2 \%$ para Cuba ${ }^{42}, 11,9 \%$ para Ecuador ${ }^{43}$ y de $5,8 \%$ para Brasil ${ }^{44}$, de manera que la prevalencia en esta población es menor a la de otros países latinoamericanos, pero en el rango de los valores citados en la guía Colombiana para otros países latinoamericanos $(4,2 \%-7,6 \%)^{9}$. 
Tabla 1. Características de la pobalción incluida

\begin{tabular}{|c|c|c|}
\hline \multicolumn{2}{|c|}{ Edad gestacional al primer control } & $\begin{array}{l}12 \pm 0.74 \\
\text { semanas de } \\
\text { gestación }\end{array}$ \\
\hline \multicolumn{2}{|c|}{ Edad materna en años al primer control } & $25 \pm 0,7$ \\
\hline \multicolumn{2}{|c|}{$\begin{array}{l}\text { Peso promedio al primer control en } \\
\text { kilogramos }\end{array}$} & $63 \pm 1.63$ \\
\hline \multicolumn{2}{|c|}{ Talla promedio al primer control en metros } & $1.56 \pm 0.71$ \\
\hline $\begin{array}{l}\text { Lugar de residencia de la } \\
\text { gestante }\end{array}$ & Frecuencia (\%) & $\begin{array}{l}\text { IC inferior - } \\
\text { superior al } \\
95 \%\end{array}$ \\
\hline Urbano & $151(52,43 \%)$ & $46,49-58,32 \%$ \\
\hline Rural & $137(47,55 \%)$ & $41,34-53,16 \%$ \\
\hline \multicolumn{3}{|c|}{ Escolaridad al primer control } \\
\hline Secundaria completa & $143(49,65 \%)$ & $43,73-55,58$ \\
\hline Secundaria en curso & $31(10,76 \%)$ & $7,43-14,93$ \\
\hline Secundaria inconclusa & $95(32,99 \%)$ & $27,58-38,74$ \\
\hline $\begin{array}{l}\text { Estudios técnicos o } \\
\text { tecnológicos }\end{array}$ & $8(2,78 \%)$ & $1,21-5,4$ \\
\hline Pregrado universitario & $5(1,74 \%)$ & $0,57-4$ \\
\hline Sin información & $5(1,74 \%)$ & $0,57-4$ \\
\hline Postgrado universitario & $1(0,35 \%)$ & $0,01-1,92$ \\
\hline \multicolumn{3}{|l|}{ VÍA DEL PARTO } \\
\hline Parto vaginal & $126(43,75 \%)$ & $37,94-49,69$ \\
\hline Cesárea & $143(49,65 \%)$ & $43,73-55,58$ \\
\hline $\begin{array}{l}\text { Perdida gestacional } \\
\text { temprana }\end{array}$ & $19(6,6 \%)$ & $4,02-10,11$ \\
\hline \multicolumn{3}{|c|}{$\begin{array}{l}\text { Clasificación del estado nutricional de la gestante en el primer } \\
\text { control prenatal }\end{array}$} \\
\hline Enflaquecida & $54(18,75 \%)$ & $14,41-23,75$ \\
\hline Normal & $115(39,93 \%)$ & $34,23-45,84$ \\
\hline Obesa & $52(18,06 \%)$ & $13,79-22,99$ \\
\hline Sobrepeso & $67(23,26 \%)$ & $18,51-28,58$ \\
\hline \multicolumn{3}{|c|}{ Antecedente personal de diabetes gestacional } \\
\hline $\begin{array}{l}\text { Antecedente personal de } \\
\text { diabetes gestacional }\end{array}$ & $9(3,13 \%)$ & $1,44-5,85 \%$ \\
\hline $\begin{array}{l}\text { Población total para las } \\
\text { variables mostradas en } \\
\text { la presente tabla }\end{array}$ & $288(100 \%)$ & \\
\hline
\end{tabular}

Fuente: autor

Despierta particular interés el hallazgo de obesidad y sobrepeso en el $83 \%$ de la población con diabetes gestacional, superior al reportado en países latinoamericanos como México $(58,4 \%)^{41}$, pero inferior a la frecuencia descrita en Cuba $(86.5 \%)^{42}$.
Tabla 2. Frecuencia de parto pretermino y trastorno hipertensivo asociado al embarazo

\begin{tabular}{|c|c|c|}
\hline & Frecuencia (\%) & $\begin{array}{c}\text { IC inferior - } \\
\text { superior al 95\% }\end{array}$ \\
\hline Parto pretérmino & $10(3.72 \%)$ & $1.46-5.98 \%$ \\
\hline $\begin{array}{c}\text { Trastorno } \\
\text { hipertensivo } \\
\text { asociado al } \\
\text { embarazo }\end{array}$ & $13(4.83 \%)$ & $2.27-7.40 \%$ \\
\hline $\begin{array}{c}\text { Total de maternas } \\
\text { empleadas para } \\
\text { la frecuencia } \\
\text { de descenlaces } \\
\text { listados. }\end{array}$ & $269(100 \%)$ & \\
\hline
\end{tabular}

Fuente: autor

Respecto a la edad materna al momento de la gestación, México describe el intervalo de edades entre 28 y 36 años como el de mayor incidencia para diabetes gestacional ${ }^{41}$, mientras que es entre $31 \mathrm{y}$ 40 años para cuba ${ }^{42}$; en ambos países, al igual que en este estudio, describen una frecuencia mayor en edades maternas más avanzadas.

Con relación a los otros factores de riesgo descritos se encontró una frecuencia alta para los trastornos hipertensivos asociados al embarazo y el trabajo de parto pretérmino, lo cual pone en evidencia la necesidad de ejecutar estudios con diseño analítico que permitan evaluar la relación de la prevalencia de diabetes con las frecuencias de los factores de riesgo en la población Colombiana, para de esta manera plantear diferentes estrategias que disminuyan su frecuencia, y con ello la prevalencia de diabetes gestacional.

Es necesario hacer la acotación de que al procurar obtener el mayor tamaño muestral posible se incluyeron en el estudio las gestantes que con más de 29 semanas asistieron a su primer control prenatal lo cual puede ser interpretado como una posible fuente de sesgo de selección, pues si bien a estas gestantes también se les realizó curva de tolerancia oral a la glucosa con un resultado negativo para el diagnóstico de diabetes gestacional, es bien sabido que los valores de los métodos diagnósticos empleados están estandarizados para su aplicación en gestantes de entre 24 y 28 semanas. Por lo que se puede presumir que a edades posteriores los criterios diagnósticos puedan tener una sensibilidad y especificidad diferentes. Fueron 12 las gestaciones 
que ingresaron a controles prenatales con 29 o más semanas de edad gestacional.

\section{Conclusiones}

La prevalencia de diabetes gestacional obtenida en el presente estudio resultó inferior a la descrita en otros estudios en Latinoamérica, sin embargo, los resultados de este estudio permiten generar una alerta sobre la frecuente presencia de malnutrición en la población en edad fértil, por lo que se deberían implementar políticas de salud pública que intervengan de manera eficiente los hábitos dietarios de la población general, para fomentar un peso saludable y de esta manera reducir la incidencia de la diabetes gestacional entre otras múltiples patologías asociadas a sobrepeso y obesidad.

\section{Consideraciones éticas}

El proyecto fue puesto a revisión y aprobado por el Comité de Ética del hospital Gestionar Bienestar en Zapatoca, Santander.

\section{Fuente de financiación}

La investigación se realizó con recursos del autor. Ninguna fuente externa patrocino el diseño ni ejecución del proyecto de investigación.

\section{Agradecimientos}

Agradecimiento al Dr. Javier Ayala Duran Director del hospital Gestionar Bienestar por haber facilitado el espacio y concedido su visto bueno para la realización de este proyecto de investigación. Igualmente debo reconocer el gran aporte recibido por la auxiliar de archivo, Doña Gloria Camelo quien con mucha paciencia y diligencia me asistió en el proceso de búsqueda de historias clínicas.

\section{Conflicto de intereses}

\section{Los autores no declaran conflicto de interés}

\section{Referencias bibliográficas}

1. Metzger BE, Buchanan TA, Coustan DR, de Leiva A, Dunger DB, Hadden DR, et al. Summary and recommendations of the Fifth International Workshop-Conference on Gestational Diabetes Mellitus. Diabetes Care [Internet]. 2007 [2018 Mar 6];30 (2):S251-60. Available from: http://www.ncbi.nlm.nih.gov/ pubmed/17596481

2. American Diabetes Association. Standards of medical care in diabetes--2012. Diabetes Care [Internet]. 2012 [2018 Mar 7];35 (1):S11-63. Available from: http://www.ncbi.nlm.nih.gov/ pubmed/22187469

3. Magee MS, Walden CE, Benedetti TJ, Knopp RH. Influence of Diagnostic Criteria on the Incidence of Gestational Diabetes and Perinatal Morbidity. JAMA [Internet]. 1993 [2018 Mar 7];269(5):609. Available from: http://jama.jamanetwork.com/ article.aspx?doi=10.1001/jama.1993.03500050087031

4. ACOG commite on practices bulletin. GESTATIONAL DIABETES . ACOG [Internet].2001 [2018 Mar 7]; 98(30). Available from: http://c.ymcdn.com/sites/chronicdisease.site-ym.com/resource/ resmgr/Womens_Health_Council_GDM/pb030_gestational_ diabetes_r.pdf

5. Micalo. T, Palay. M GM. Diabetes gestacional: diagnóstico y manejo. JANO EMC. 1998;55(1271):64-71.

6. Centers for Disease Control and Prevention. National diabetes fact sheet: national estimates and general information on diabetes and prediabetes in the United States, 2011. Atlanta, GA: U.S. Department of Health and Human Services, Centers for Disease Control and Prevention, 2011.

7. Jiwani A, Marseille E, Lohse N, Damm P, Hod M, Kahn JG. Gestational diabetes mellitus: results from a survey of country prevalence and practices. J Matern Neonatal Med [Internet]. 2011 [cited 2018 Mar 7];25(6):600-10. Available from: http://www. tandfonline.com/doi/full/10.3109/14767058.2011.587921

8. Kampmann U, Madsen LR, Skajaa GO, Iversen DS, Moeller N, Ovesen P. Gestational diabetes: A clinical update. World J Diabetes [Internet]. 2015 [cited 2018 Mar 7];6(8):1065-72. Available from: http://www.ncbi.nlm.nih.gov/pubmed/26240703

9. Colciencias. Guía de práctica clínica para el diagnóstico, tratamiento y seguimiento de la diabetes Gestacional Sistema General de Seguridad Social en Salud - Colombia. Minist Salud y Protección Soc [Internet]. 2016 [cited 2018 Mar 7]; Available from: http://gpc.minsalud.gov.co/gpc_sites/Repositorio/Conv_637/ GPC diabetes/DIABETES GESTACIONAL COMPLETA.pdf

10. Ferrara A. Increasing prevalence of gestational diabetes mellitus: a public health perspective. Diabetes Care [Internet]. 2007 [cited 2018 Mar 7];30 (Suppl 2):S141-6. Available from: http://www. ncbi.nlm.nih.gov/pubmed/17596462

11. Burbano-López RM, Castaño-Castrillón JJ, González-Castellanos L, González-Henao HS, Quintero-Ospina JD, Revelo-Imbacuan LDJ, et al. Frecuencia de Diabetes Mellitus Gestacional y factores de riesgo en gestantes atendidas en clínicas de ASSBASALUD ESE, Manizales (Colombia) 2011-2012: estudio de corte transversal. Rev Colomb Obstet Ginecol [Internet]. 2014 [citado 7 Mar 2018];65(4):338-45. Disponible en: http://revista.fecolsog. org/index.php/rcog/article/view/38

12. García F, Solís J, Calderón J, Luque E, Neyra L, Manrique H, et al. Prevalence of diabetes mellitus and related risk factors in an urban population. Rev Soc Peru Med Interna [Internet]. 2007 [cited 2018 Mar 7];20(3):90-4. Available from: http:// medicinainterna.org.pe/revista/revista_20_3_2007/3.pdf

13. ALAD. Guías ALAD de diagnóstico, control y tratamiento de la diabetes mellitus tipo 2. Epidemiología de la diabetes mellitus en Latinoamérica. Rev la Asoc Latinoam Diabetes. 2000;Supl No 1:116-9.

14. Campo MZ, Estrada G. Factores de riesgo para Diabetes Gestacional en población obstétrica. CES Med [Internet]. 2008 [citado 7 Mar 2018];22(1):59-69. Available from: http://revistas. ces.edu.co/index.php/medicina/article/view/525

15. Contreras-Zúñiga E, Guillermo-Arango L, Zuluaga-Martínez SX, Ocampo V. DIABETES Y EMBARAZO. Rev Colomb Obstet Ginecol [Internet]. 2008 [citado 7 Mar 2018];59(1):38-45. Disponible en: http://www.scielo.org.co/pdf/rcog/v59n1/v59n1a06.pdf

16. Lindström J, Louheranta A, Mannelin M, Rastas M, Salminen V, Eriksson J, et al. The Finnish Diabetes Prevention Study (DPS): Lifestyle intervention and 3-year results on diet and physical activity. Diabetes Care [Internet]. 2003 [cited $2018 \mathrm{Mar}$ 7];26(12):3230-6. Available from: http://www.ncbi.nlm.nih.gov/ pubmed/14633807

17. Di Cianni G, Miccoli R, Volpe L, Lencioni C, Del Prato S. Intermediate metabolism in normal pregnancy and in gestational diabetes. Diabetes Metab Res Rev [Internet]. 2003 [cited 2018 Mar 7];19(4):259-70. Available from: http://doi.wiley.com/10.1002/ 
dmrr.390

18. García CG. Diabetes mellitus gestacional. Med Int México Vol [Internet]. 2008 [citado 7 Mar 2018];24(2):148-56. Disponible en: http://www.medigraphic.com/pdfs/medintmex/mim-2008/ mim082h.pdf

19. Li Y, Hadden C, Singh P, Mercado CP, Murphy P, Dajani NK, et al. GDM-associated insulin deficiency hinders the dissociation of SERT from ERp44 and down-regulates placental 5-HT uptake. Proc Natl Acad Sci U S A [Internet]. 2014 [cited 2018 Mar 7];111(52):E5697-705. Available from: http://www.ncbi.nlm.nih. gov/pubmed/25512553

20. Corcoy R, Lumbreras B, Bartha JL, Ricart W, Grupo Español de Diabetes y Embarazo. New diagnostic criteria for gestational diabetes mellitus after the HAPO study. Are they valid in our environment? Gac Sanit [Internet]. 2010 [cited 2018 Mar 7];24(4):361-3. Available from: http://scielo.isciii.es/scielo. php?script $=$ sci arttext\&pid $=$ S0213-91112010000400017

21. Flores-Padilla L, Solorio-Páez IC, Melo-Rey ML, Trejo-Franco J. Embarazo y obesidad: riesgo para desarrollo de diabetes gestacional en la frontera norte de México Gac. Méd. Méx [Internet]. 2014 [citado 1 abril 2018]; 150(1):73-8. Disponible en: https://www.anmm.org.mx/GMM/2014/s1/GMM_150_2014 S1_073-078.pdf

22. Lozano A, Betancourth WR , Turcios LJ, Ocampo DM, Portillo CV, Lozano L. Sobrepeso y Obesidad en el Embarazo: Complicaciones y Manejo.Archivos de medicina [Internet]. 2016 [consultado 1 abril 2018]; 12(3):11. Disponible en: http://www. archivosdemedicina.com/medicina-de-familia/sobrepeso-yobesidad-en-el-embarazo-complicaciones-y-manejo.pdf

23. England LJ, Dietz PM, Njoroge T, Callaghan WM, Bruce C, Buus RM, et al. Preventing type 2 diabetes: public health implications for women with a history of gestational diabetes mellitus. Am J Obstet Gynecol [Internet]. 2009 [citado 7 marzo 2018]; 200(4):365.e1-365.e8. Disponible en: http://linkinghub.elsevier. com/retrieve/pii/S000293780800642X

24. Committee Opinion No. 504. Obstet Gynecol [Internet]. 2011 [citado 7 marzo 2018]; 118(3):751-3. Disponible en: http://www. ncbi.nlm.nih.gov/pubmed/21860317

25. Hedderson MM, Ferrara A, Sacks DA. Gestational diabetes mellitus and lesser degrees of pregnancy hyperglycemia: association with increased risk of spontaneous preterm birth. Obstet Gynecol [Internet]. 2003 [citado 7 marzo 2018]; 102(4):850-6. Disponible en: http://www.ncbi.nlm.nih.gov/pubmed/14551018

26. Ostlund I, Haglund B, Hanson U. Gestational diabetes and preeclampsia. Eur J Obstet Gynecol Reprod Biol [Internet]. 2004 [citado 7 marzo 2018]; 113(1):12-6. Disponible en: http://www. ncbi.nlm.nih.gov/pubmed/15036703

27. Kaaja RJ, Greer IA. Manifestations of Chronic Disease During Pregnancy. JAMA [Internet]. 2005 [citado 7 marzo 2018]; 294(21):2751. Disponible en: http://jama.jamanetwork.com/ article.aspx?doi=10.1001/jama.294.21.2751

28. Buchanan TA, Xiang AH. Gestational diabetes mellitus. J Clin Invest [Internet]. 2005 [citado 7 marzo 2018]; 115(3):485-91. Disponible en: http://www.ncbi.nlm.nih.gov/pubmed/15765129

29. Han S, Middleton PF, Bubner TK, Crowther CA. Women's Views on Their Diagnosis and Management for Borderline Gestational Diabetes Mellitus. J Diabetes Res [Internet]. 2015 [citado 7 marzo 2018]; 2015:1-9. Disponible en: http://www.hindawi.com/ journals/jdr/2015/209215/

30. Albareda M, Caballero A, Badell G, Piquer S, Ortiz A, de Leiva $\mathrm{A}$, et al. Diabetes and abnormal glucose tolerance in women with previous gestational diabetes. Diabetes Care [Internet]. 2003 [citado 7 marzo 2018]; 26(4):1199-205. Disponible en: http:// www.ncbi.nlm.nih.gov/pubmed/12663597

31. Lee AJ, Hiscock RJ, Wein P, Walker SP, Permezel M. Gestational diabetes mellitus: clinical predictors and long-term risk of developing type 2 diabetes: a retrospective cohort study using survival analysis. Diabetes Care [Internet]. 2007 [citado 7 marzo 2018]; 30(4):878-83. Disponible en: http://www.ncbi.nlm.nih. gov/pubmed/17392549

32. Kim C, Newton KM, Knopp RH. Gestational diabetes and the incidence of type 2 diabetes: a systematic review. Diabetes Care [Internet]. 2002 [citado 7 marzo 2018]; 25(10):1862-8. Disponible en: http://www.ncbi.nlm.nih.gov/pubmed/12351492

33. Hedderson MM, Weiss NS, Sacks DA, Pettitt DJ, Selby J V, Quesenberry CP, et al. Pregnancy Weight Gain and Risk of Neonatal Complications. Obstet Gynecol [Internet]. 2006 [citado 7 mazo 2018]; 108(5):1153-61. Disponible en: http://www.ncbi. nlm.nih.gov/pubmed/17077237

34. Ríos-Martínez W, García-Salazar A, Ruano-Herrera L, EspinosaVelasco M, Zárate A, Hernández-Valencia M. Complicaciones obstétricas de la diabetes gestacional: criterios de la IADPSG y HAPO. Perinatol Reprod Hum [Internet]. 2013 [citado 7 marzo 2018]; 28(1):27-32. Disponible en: http://www.medigraphic.com/ pdfs/inper/ip-2014/ip141e.pdf

35. Dabelea D, Hanson RL, Lindsay RS, Pettitt DJ, Imperatore G, Gabir MM, et al. Intrauterine exposure to diabetes conveys risks for type 2 diabetes and obesity: a study of discordant sibships. Diabetes [Internet]. 1 de diciembre de 2000 [acceso 7 de marzo de 2018];49(12):2208-11. Available from: https://www.ncbi.nlm. nih.gov/pubmed/11118027

36. Catalano PM, Kirwan JP, Haugel-de Mouzon S, King J. Gestational diabetes and insulin resistance: role in short- and long-term implications for mother and fetus. J Nutr [Internet]. 2003 mayo [acceso 7 de marzo de 2018];133(5):1674S-83S. Available from: http://www.ncbi.nlm.nih.gov/pubmed/12730484

37. Dabelea D, Mayer-Davis EJ, Lamichhane AP, D'Agostino RB, Liese AD, Vehik KS, et al. Association of intrauterine exposure to maternal diabetes and obesity with type 2 diabetes in youth: the SEARCH Case-Control Study. Diabetes Care [Internet]. 1 de julio de 2008 [acceso 7 de marzo de 2018];31(7):1422-6. Available from: http://www.ncbi.nlm.nih.gov/pubmed/18375420

38. International Association of Diabetes and Pregnancy Study Groups Consensus Panel IA of D and PSGC, Metzger BE, Gabbe SG, Persson B, Buchanan TA, Catalano PA, et al. International association of diabetes and pregnancy study groups recommendations on the diagnosis and classification of hyperglycemia in pregnancy. Diabetes Care [Internet]. marzo 2010 [acceso 9 de marzo de 2018];33(3):676-82. Available from: http://www.ncbi.nlm.nih.gov/pubmed/20190296

39. Atalah E, Castillo C, Castro R AA. Propuesta de un nuevo estandar de evaluacion nutricional en embarazadas. Rev Méd Chile [Internet]. 1997 [acceso 9 de marzo de 2018];125:142936. Available from: https://www.researchgate.net/profile/ Eduardo_Atalah/publication/13675304_Proposal of a new standard_for_the_nutritional_assessment_of_pregnant_women/ links/543c314f0cf24ef33b757538/Proposal-of-a-new-standardfor-the-nutritional-assessment-of-pregnant-women

40. Institute of medicine. Nutrition During Pregnancy: Part I: Weight Gain, Part II: Nutrient Supplements [Internet]. Washington, D.C.: National Academies Press; 1990 [acceso 9 de marzo de 2018]. Available from: http://www.nap.edu/catalog/1451

41. González Ruiz MN, Rodríguez Bandala C, Salcedo Vargas M, Martínez Lara E, Enríquez Espinoza F, Polo Soto SM, et al. Actualidades en diabetes gestacional. Rev Sanid Milit Mex [Internet]. 2014 [acceso 9 de marzo de 2018];68(5):276-82. Available from: http://www.medigraphic.com/pdfs/sanmil/sm2014/sm145f.pdf

42. Casas-Lay Y, Sánchez-Salcedo M, Álvarez-Rodríguez JM. Algunas variables epidemiológicas en pacientes con diabetes mellitus gestacional. Rev Cubana Obstet Ginecol. 2014; 40(1): 2-12.

43. Jiménez WJ. Prevalencia de diabetes gestacional y factores de riesgo en pacientes del Hospital Maternidad Enrique C. Sotomayor Guayaquil 2009-2011 [master's thesis]. Guayaquil, EC; Universidad de Guayaquil; 2015. 122 p.

44. Massucatti LA, Pereira RAP, Maioli TU. Prevalência de diabetes gestacional em Unidades de Saúde Básica. Rev Enferm Atençao Saude. 2012;1(1): 70-9. 